\title{
Hybrid Camera and Real-View Thermography for Nondestructive Evaluation
}

\author{
by L. Bienkowski*, C. Homma*, K. Eisler*, C. Boller** \\ *Corporate Research \& Technologies, Siemens AG, Otto-Hahn-Ring 6, 81730 Munich, \\ christian.homma@siemens.com \\ **Fraunhofer IZFP Saarbrücken, Institut für Zerstörungsfreie Prüfverfahren, Campus, Geb. E3.1, 66123 Saarbrücken
}

\begin{abstract}
In this paper we present two novel approaches for enhancing active thermography for nondestructive testing: the hybrid camera setup is used to merge IR images with additional color images to provide the inspector with additional information on the screen. In order to make the evaluation of the data even more intuitive a real-view setup is presented that uses a projection technique to let the inspector view and interact with the measurement results directly on the part in a very intuitive way.
\end{abstract}

\section{Introduction}

Today active thermography is a widely used imaging non-destructive testing technique. However, for complex parts the evaluation can still be cumbersome, e. g. when trying to identify false calls in the result image or when locating defects on the real part. We propose two technologies: the first merges an IR image with a color picture (Hybrid camera) and the second projects the inspection results onto the part (Real-View thermography).

\section{Hybrid camera}

The hybrid camera consists of two individual cameras sensitive in different wavelength ranges that share the same beam path by using a beam splitter [1-4]. In principle any combination of spectral ranges can be used, e. $g$. MWIR/LWIR (mid-wave infrared/long-wave infrared) or VIS/MWIR (visual/mid-wave infrared). We chose the variant VIS and MWIR camera. The mid-wave IR camera is a Flir SC7600, which is a standard camera for active thermography applications. However, as in most infrared cameras, the resolution is limited, in this case to $640 \times 512$ pixels. Another drawback is that the infrared image resembles average information over the wavelength range from 3 to 5 microns and is thus rather comparable to former black/white than to modern color cameras. In order to obtain more information about an object than just the infrared one, an additional color image can be used.

Such a combined imaging system is realized in the hybrid camera described here which uses a beam splitter transparent for infrared light and reflecting in the visible to combine the images of an IR and a VIS camera. Though in some IR cameras available on the market a VIS camera is already built-in, those systems have the VIS lens located next to the IR lens which results in different angles of view to the test specimen $[5,6]$. This, however, is disadvantageous for short working distances where the parallax influence can no longer be neglected and the images are not aligned anymore. Using the beam-splitter set-up the combination of IR and VIS is completely parallax-free and the highly accurate mapping of the lower resolved IR image with 640x512 pixels in the VIS picture with $1600 \times 1200$ pixels is not very demanding on the resources of the computing system. When the optical system is properly adjusted the IR picture is just scaled and translated to fit into the RGB picture without the additional use of image rotating or straightening algorithms. So the hybrid camera realizes well matched information of the IR to the VIS even at shortest working distances. Therefore the VIS information can be used e. g. to accentuate or suppress information in the IR or vice versa.

\subsection{Setup and benefit of the hybrid camera}

The described hybrid camera combines two cameras working in different wavelength ranges (Fig. 1 left). Due to the accurate mapping of the IR picture to the VIS picture and because of the significantly higher resolution of the VIS camera, it is easier to localize (Fig. 1 right and Fig. 2 left) and identify (Fig. 2 right) the source of a thermal signal. By comparing the actual assessment of defects using only the IR data with the evaluation that can be achieved by using the hybrid camera, the benefits are evident as can be seen from Fig. 2 (right). 


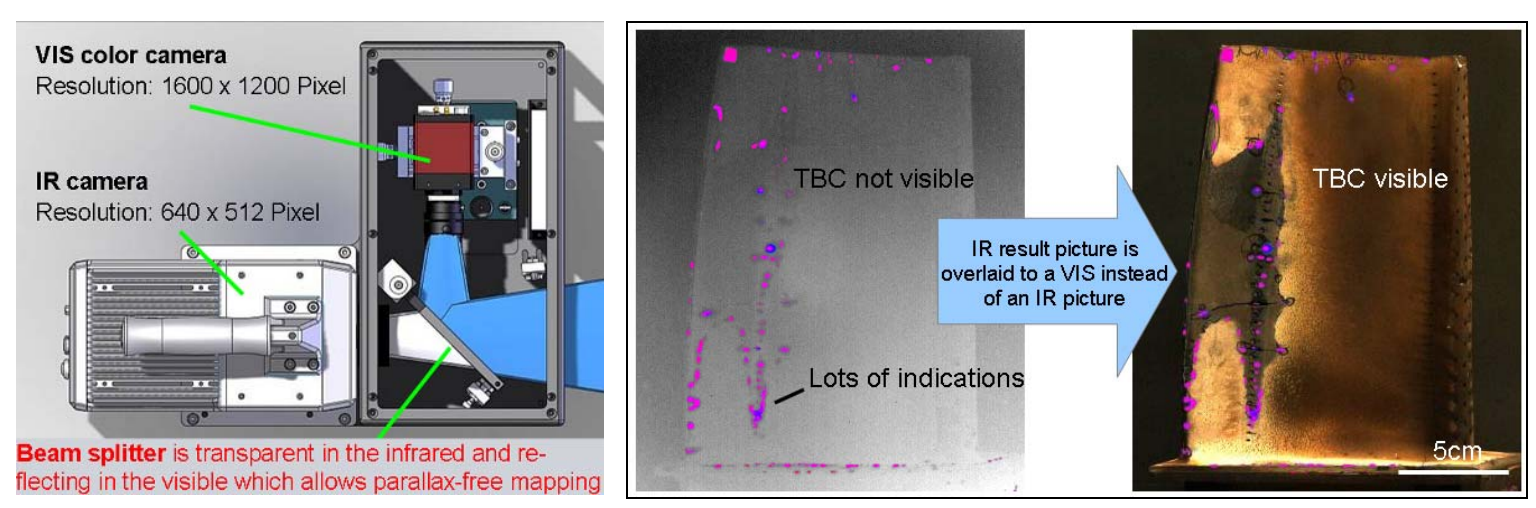

Fig. 1 Left: Schematic view of the hybrid camera with IR und VIS camera. A parallax-free combination of both wavelength regions is guaranteed by the beam combination system with proper mechanical adjustment.

Right: Conventional view of an acoustic thermography measurement result as color-coded pulse-phase image (in magenta and blue) [7] overlaid with the IR live picture. The result shows delamination of the zirconium oxide (ZrO) coating and narrow cracks. With the hybrid camera the same sonic IR result can be overlaid with a VIS picture. Thus the overall state of the object under test including cracks, color and coating is visible.
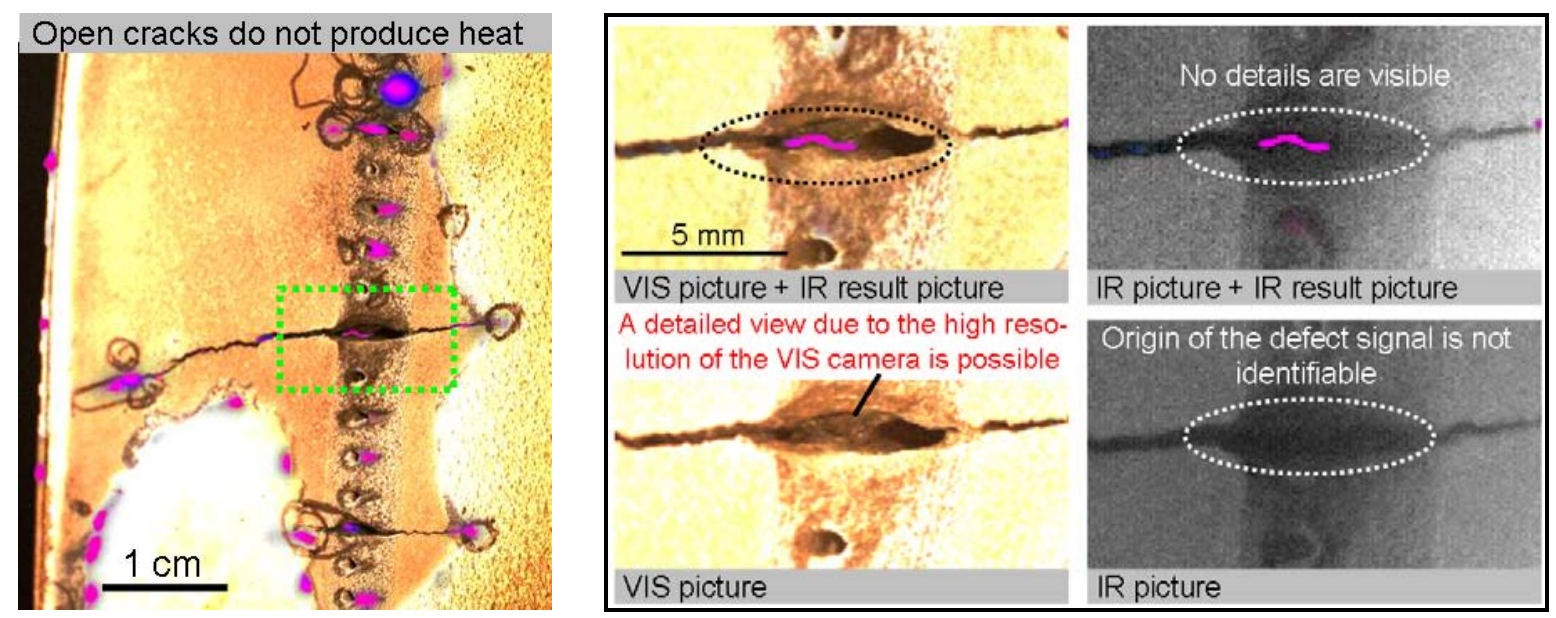

Fig. 2. Left: Acoustic thermography picture in magenta and blue of a turbine blade overlaid with the VIS picture. Right: Enlarged views of the green region of interest in the left picture. The comparison between left (hybrid image) and right (IR image) shows the benefit of the hybrid camera, e.g. a higher resolved VIS image and better contrast compared to the IR picture.

In the hybrid picture the edges of the coating, the cracks and also the color of the ZrO coating can be seen. With the high resolution of the VIS camera, it is also easier to study the heating mechanism, which has been a research topic for many years [7-11] through the parallax-free view of both cameras which allows short working distances as demonstrated in Fig. 2 (right). The actual heat source deep inside the crack is only visible in the color picture. Furthermore, because of the accuracy of the mapping the information of the VIS picture can be used to accentuate or suppress information in the corresponding IR picture.

\subsection{Hybrid camera in passive thermography}

Though this paper concentrates on active thermography, it should still be mentioned that the hybrid camera can also be used for passive thermography applications like surveillance or process monitoring. One example is shown in Fig. 3 where it is also evident that both images are captured synchronously since one camera triggers the other which results in both images fitting temporally as well as spatially. 


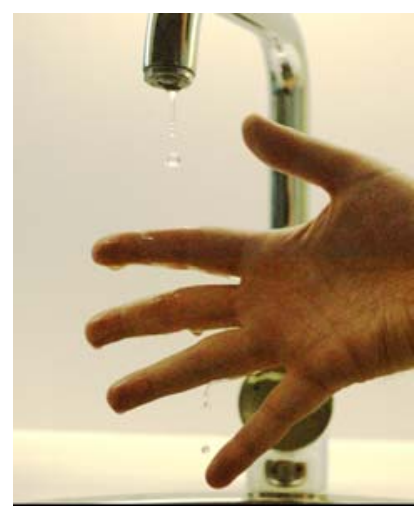

a) Just VIS image

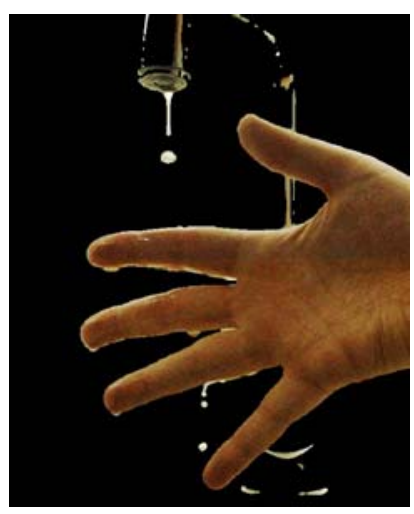

b) VIS image above IR threshold, black otherwise, i. e. segmentation using IR information

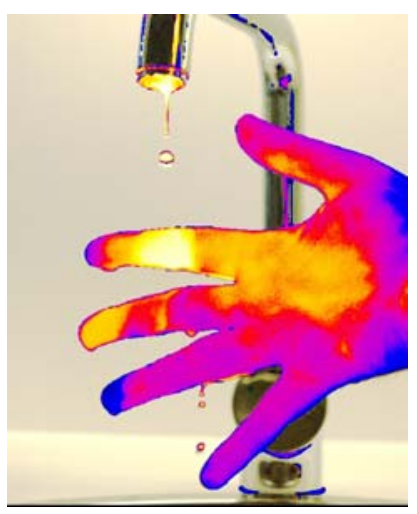

c) IR image above IR threshold, i. e. hot

information in IR, other information in VIS

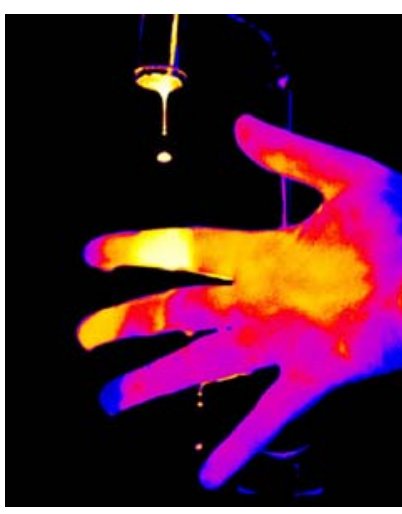

d) Just IR image

Fig. 3. Examples of visualization modes of the hybrid camera for passive thermography

\section{Real-View thermography}

\subsection{Introduction}

The result of the thermography inspection is available directly after the measurement and can be viewed on a computer screen, e. g. as color coded pulse-phase image [10]. However, even when using the hybrid camera, a comparison between the result image and the real specimen can only be done indirectly. For this reason a reliable analysis of the results is often cumbersome.

We propose a Real-View thermography system with a video projector using the specimen as a non-planar projection surface. The thermography result image is dynamically adjusted to the size as well as pose of the specimen which allows projecting the result onto it, so that the projection perfectly merges with the object features.

Two scenarios are covered by our system that will be discussed in the following sections:

1. Static case: the specimen is fixed in the inspection equipment and not moveable. To avoid any parallax error IR camera and video projector share the same optical axis using a beam splitter. Therefore no deformation of the image prior to the projection is required.

2. Dynamic case: the specimen can be moved with six degrees of freedom within a confined workspace. The position of the object is continuously tracked, so a pose matrix is available at any time. With the help of this data a geometrical correction and thus a proper projection in real-time is possible. This can be seen as an enhancement of the static case, since the specimen can be released from the inspection equipment after the measurement for better evaluation.

\subsection{Static case}

Our proposed setup for the static case is similar to the hybrid camera setup (Fig. 1 left) but uses a video projector instead of the VIS camera as can be seen in Fig. 4. This guarantees a good alignment of the projected image with the object since no parallax effects occur. 


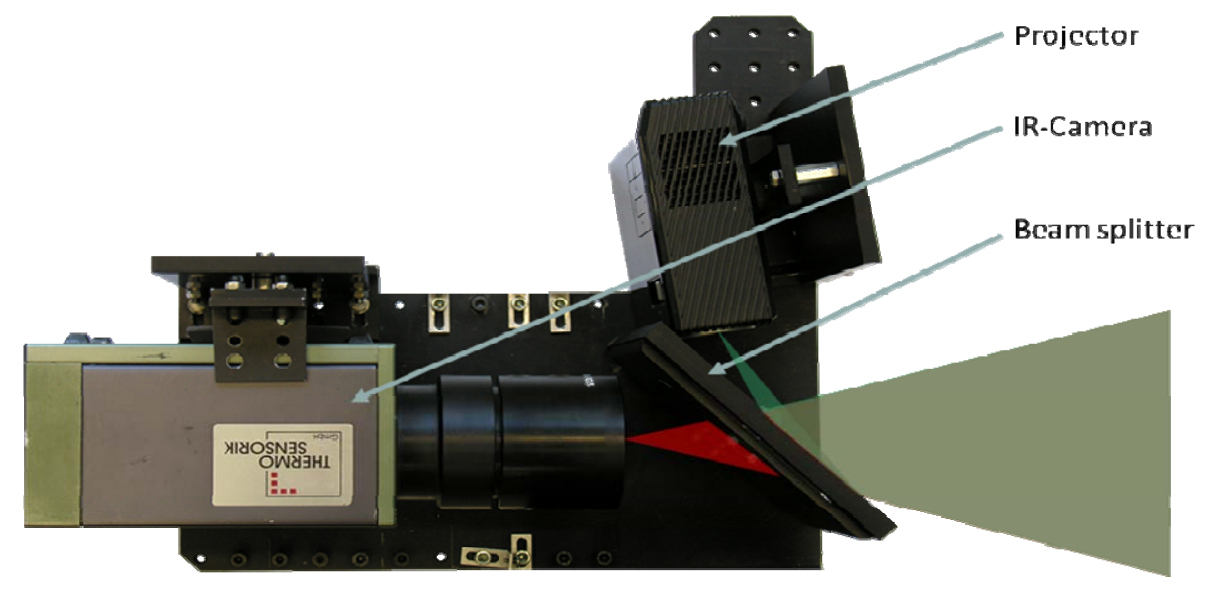

Fig. 4. View of Real-View thermography setup for the static case.

\subsubsection{Pre-measurement guidance}

The Real-View concept cannot only be used for evaluating defects but also serves as valuable guidance before the actual measurement. In a concept study we show possible information useful for part handling and starting of a measurement (cv. Fig. 5).

In a first step the part under test is being detected. All subsequent steps can then be tailored to that part type, e. g. measurement parameters, positioning, and evaluation criteria. Now hints how to position the part are given in a visual manner by arrows until the proper position is reached. Afterwards the selection of the test method is done by using the part itself as virtual touch-screen. A list of appropriate and available technologies is displayed and the inspector selects the optimum one by "clicking" on it which is realized either by a hand gesture or by means of a 3D sensor like the Microsoft Kinect. Afterwards the measurement starts and the recorded infrared data is projected on the part in real-time to give a visual feedback of the development of the IR signal.

After the measurement the data is post-processed, again utilizing the information about the part type, and the result data is accurately displayed on the part for final evaluation.

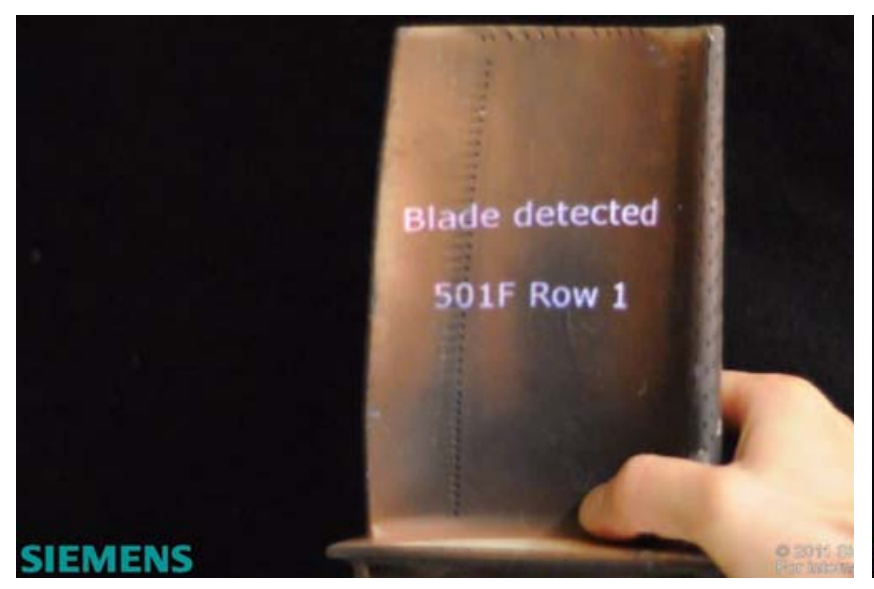

a) Automatic detection of part

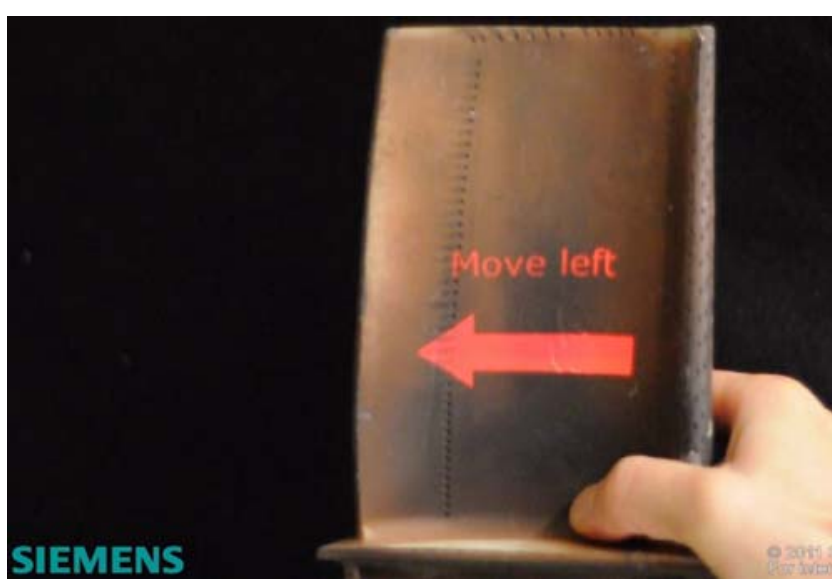

b) Hint for correct positioning 


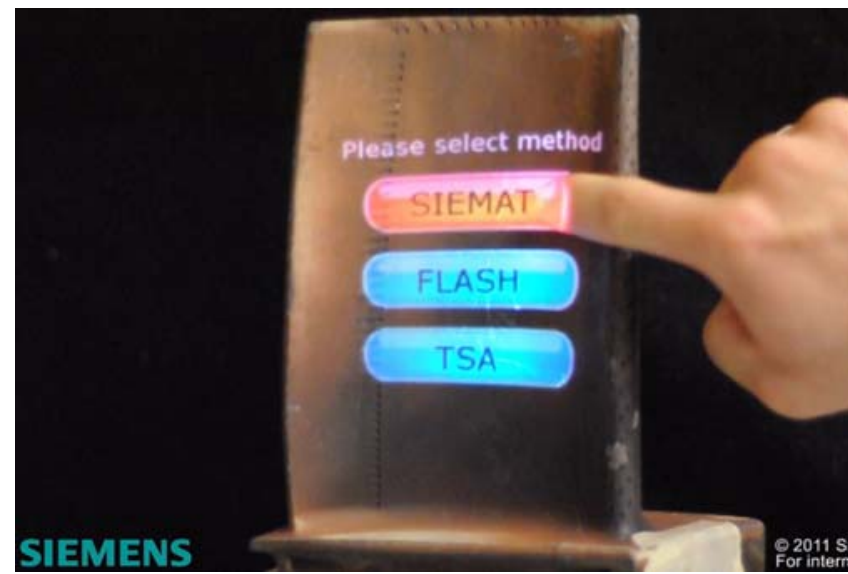

c) Selection of test method

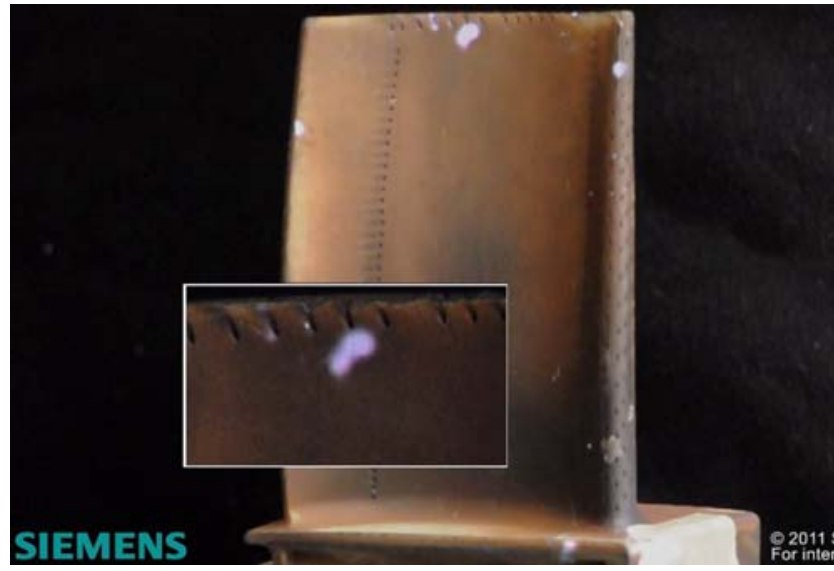

d) Live projection of infrared data (the zoomed-in inset is only shown for comparison here and not projected)

Fig. 5. Real-View concept during part handling and measurement

\subsubsection{Post-measurement interaction modes}

For working with the data on the part a pointer is tracked with the infrared camera. In the case shown it is a screwdriver, but in principle it can be any object with clearly defined contours. With the beam splitter approach no 3D detection of the pointer is necessary. If it is held reasonably close to the object the detection can be done on the 2D infrared image by simple difference and threshold detection. It is not necessary to make contact with the part, so sensitive surfaces are not damaged. The following interaction modes were implemented for the concept study (cv. Fig. 6).

\section{Detailed information}

By moving the pointer close to an indication, further information can be displayed that is compiled by one or more post-processing algorithms. Usually a defect classification would be the first step and a subsequent detailed analysis of the defect will yield additional information about its size or criticality.

\section{Magnifier}

Especially for small indications, a zoomed-in view is beneficial. In this application a magnifying glass cannot be used, since the information to be magnified is not a property of the object but just a projection. Therefore the magnification of the result image is done on the computer and the result is projected at the proper location.

\section{Virtual flashlight}

Those familiar with fluorescent penetrant inspection know that an inspector regularly switches from black to white light to evaluate indications. Under black light only the fluorescing indications can be seen while real part features are only visible in white light. A similar approach is used in Real-View Thermography where surface features might be masked by the colored projection. In order to see "through" the projection onto the real part a white area is projected that can be moved by means of the pointer.

\section{Eraser}

By using magnifier and virtual flashlight and taking the automatic detection into account, the inspector can decide if a given indication is a crack or only a false call. For acoustic thermography the latter can be caused by viscoelastic material that heats up by dampening the ultrasonic wave, e.g. organic residues [7, 12]. Those false calls might distract any further inspection and thus might not need to be archived. Therefore they can be erased by simply swiping the pointer over them. 


\section{Drawing}

Often annotations need to be made like arrows pointing to especially critical defects or circles around areas of interest. This can be achieved by switching to drawing mode and using the pointer as pencil. The modified image is then stored together with the original data for later usage.

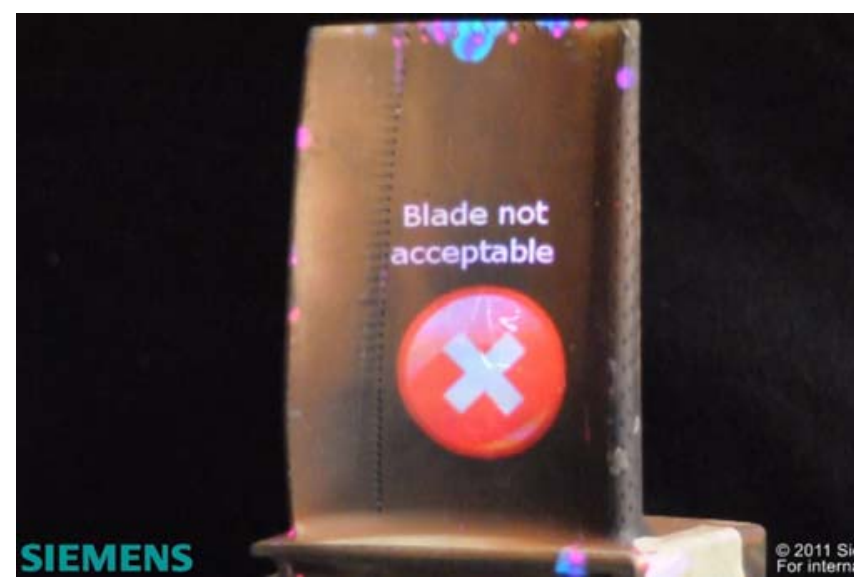

a) Automatic evaluation

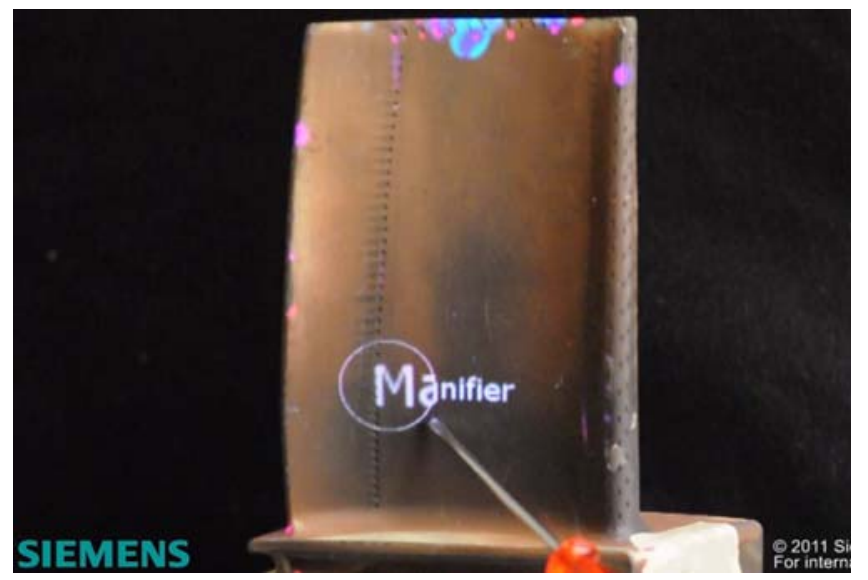

c) Magnifier

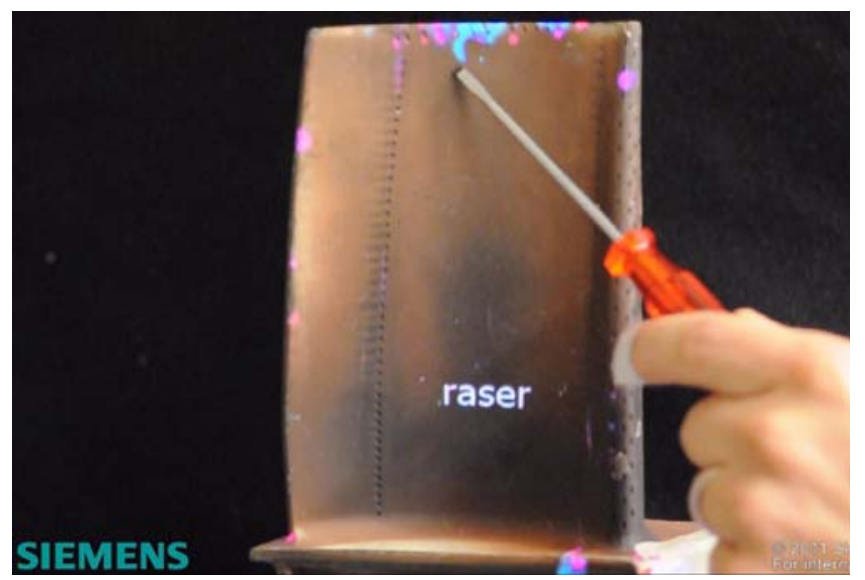

e) Eraser

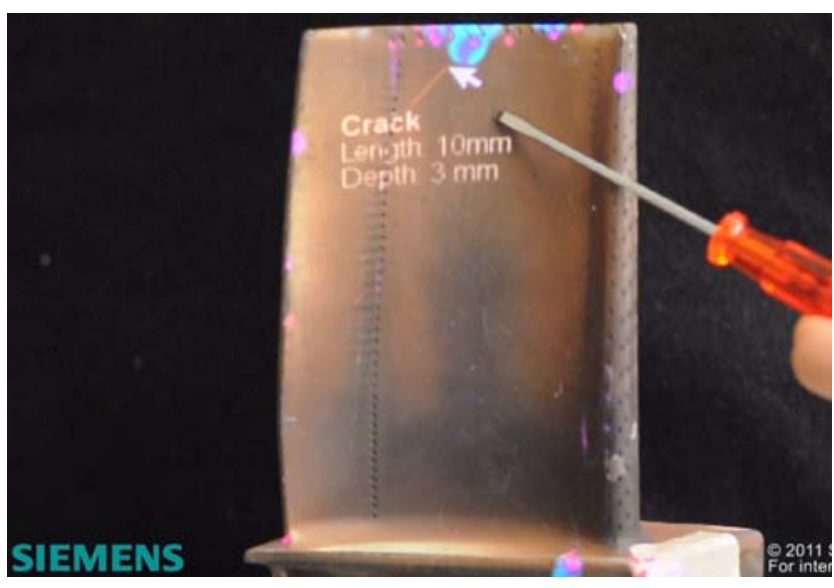

b) Detailed information about indications

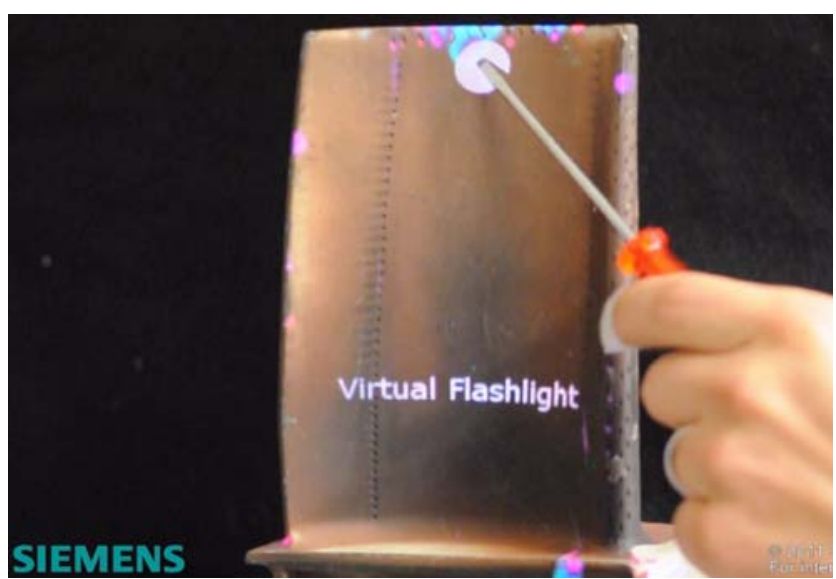

d) Virtual flashlight

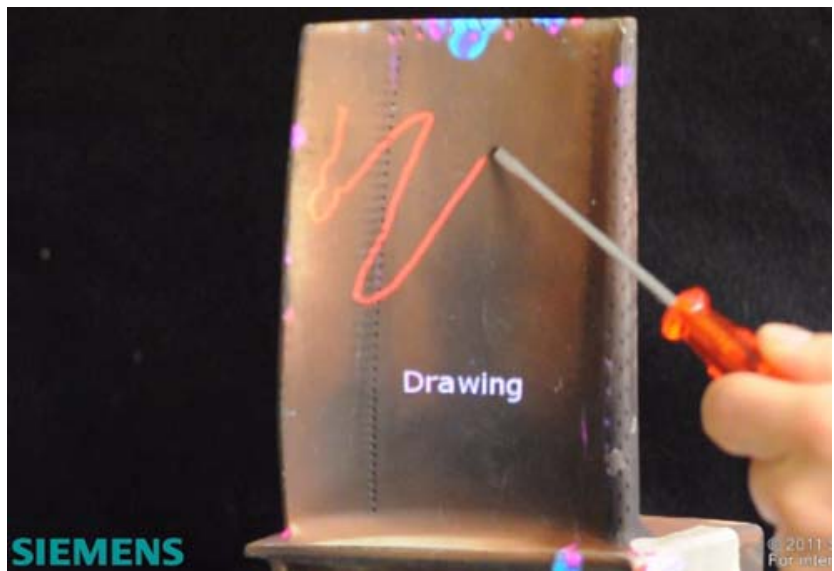

f) Drawing 
Fig. 6. Automatic evaluation of defects and different interaction modes shown on a turbine blade

\subsection{Dynamic case}

In this case the specimen can be removed from the fixture and moved to another position which is more beneficial for the projection light condition. The specimen is continuously tracked by the tracking unit (Fig. 7), which is calibrated with the video projector. Thus the position of the tracked object can be obtained and converted into the projector coordinate system.

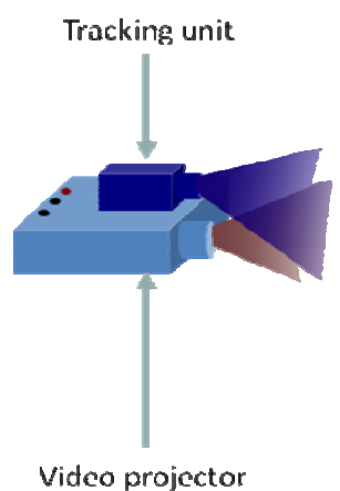

Video projector

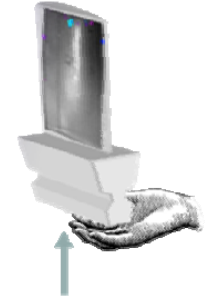

Moveable specimen

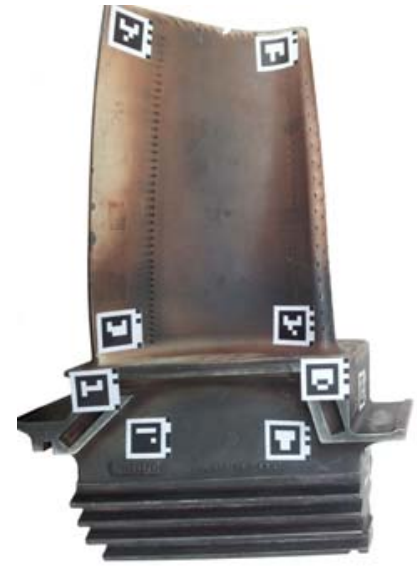

Fig. 7. Left: Schematic view of Real-View setup for dynamic case. The position of the specimen is provided by the tracking unit. Right: View of the turbine blade with markers used for marker-based tracking.

\subsubsection{Tracking}

At the moment binary coded markers are placed at known positions on the specimen (Fig. 7 right). The tracking unit captures images of the scene, which are subsequently analyzed by a standard marker finder algorithm. At least two markers must be found to calculate the pose (rotation and translation in the tracking unit coordinate system) of the tracked object. In addition during the tracking step the video projector must be inactive e. g. by projecting a black image, to avoid errors in the marker recognition.

In future we will follow two strategies: a 2D texture based marker-less tracking as well as a 3D tracking using 3D cameras like the Microsoft Kinect. Though these techniques differ in the way the pose matrix is acquired, the projection technology is the same as explained in the next section.

\subsubsection{Geometric adjustments of the result image}

Since the specimen that is the projection plane is moveable and usually non-planar, it is necessary to apply geometric compensation to the image before projection, since in the dynamic case the camera and projector are not aligned collinear in contrast to the static case. To achieve this, the result image is processed after the measurement and mapped on the 3D model of the inspected specimen as a texture (as shown in Fig. 8 left). This occurs only once to complete the initial registration. Afterwards the current pose of the specimen is obtained continuously. The 3D model with the mapped texture is then moved and rotated virtually according to the acquired pose. The position of the virtual camera in the 3D space in relationship to the model is identical with this of the video projector in real world. So the view generated by the virtual camera can be used as a projection output (Fig. 8 right). The projected image aligns perfectly with the specimen.

Both the tracking and the geometric adjustment are fast enough to give an impression of smoothness during the evaluation. 

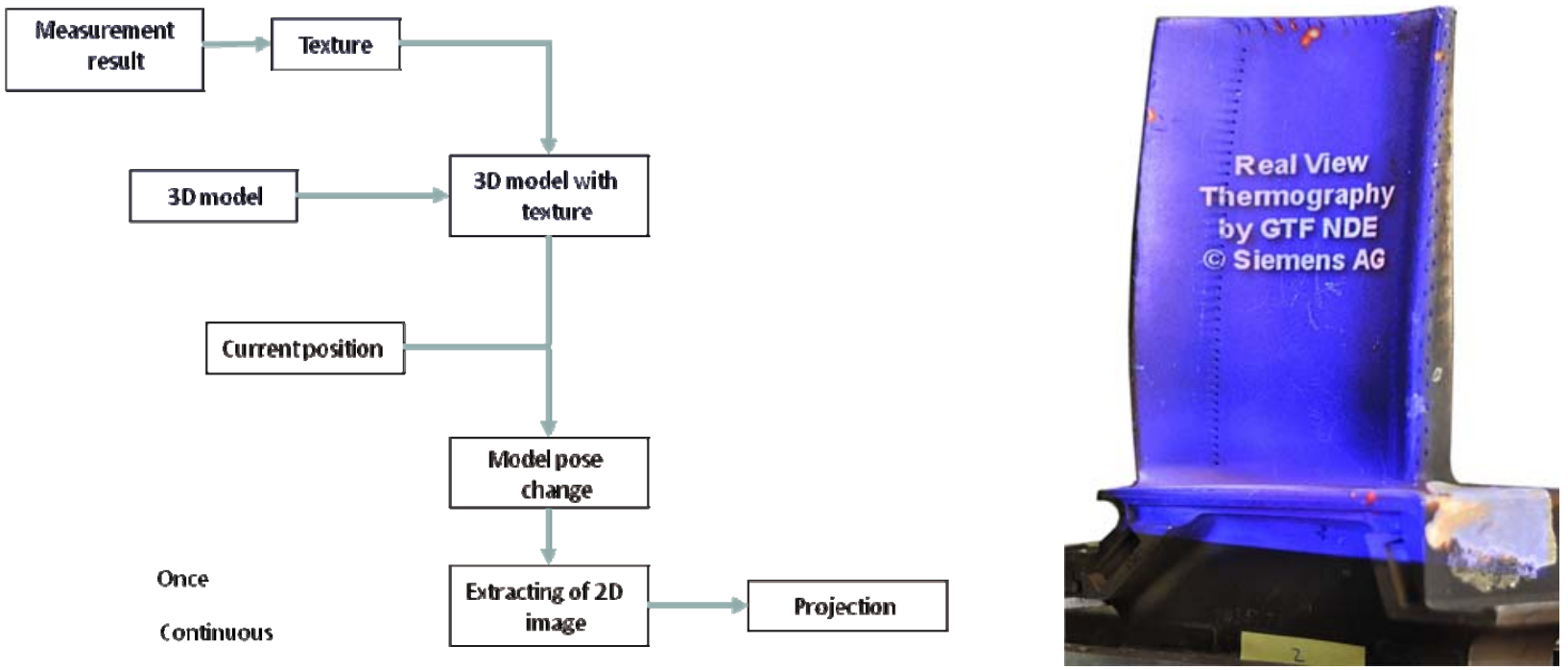

Fig. 8. Left: The schematic view of the geometrical image adjustment algorithm.

Right: Projection of the geometrically adjusted thermography result image on the real turbine blade.

\section{Summary}

In this paper we presented two technologies to overcome current limitations of active thermography to make it fit into existing applications more easily and potentially replace other methods like penetrant testing. Both methods help making thermography even more intuitive: the hybrid camera gives the inspector a standard visual image at hand which is a view he is usually more acquainted with than with the IR image. This allows aligning features in both images more easily and therefore improves interpretation of thermography indications. The Real-View set-up, on the other hand, takes this concept a step further by projecting the information directly on the part to further enhance evaluation. Next steps include the full implementation of the dynamic case with marker-less tracking as well as identification of a specific test part in a group of parts, e. g. when a whole set of blades is stored at a warehouse and the individual results for each part shall be projected. Given the reduction in size of current projectors, even a handheld device seems in reach.

\section{REFERENCES}

[1] Eisler, K.; Goldammer, M.; Rothenfusser, M.; Homma, C.; Arnold, W.: „Wie bringt man Farbe in die Thermografie?" In: Deutsche Gesellschaft für zerstörungsfreie Prüfung e. V. (Berlin) - Jahrestagung, 2010. Berichtsband 122-CD, Poster 38

[2] Eisler, K.; Goldammer, M.; Rothenfusser, M.; Arnold, W.; Homma, C.: „Parallaxenfreie Bildfusion des infraroten und des sichtbaren Spektralbereichs ohne Kenntnis der 3D-Oberflächeninformation“. In: Deutsche Gesellschaft für zerstörungsfreie Prüfung e. V. (Berlin) - Jahrestagung, 2011. - Berichtsband 127-CD, Poster 48

[3] Eisler, K.;Goldammer, M.; Rothenfusser, M.; Arnold, W.; Homma, C.: "Combining Spectral Material Properties in the Infrared and the Visible Spectral Range for Qualification and Nondestructive Evaluation of Components". In: Thompson, D. O.; Chimenti, D. E. (Hrsg.): AIP Conference Proceedings-Review of Quantitative Nondestructive Evaluation, 2011.

[4] Eisler, K.; Goldammer, M.; Rothenfusser, M.; Arnold, W.; Homma, C.: „Nutzung der multispektralen Eigenschaften von Materialien zur Qualifizierung und zerstörungsfreien Prüfung von Komponenten." In: Deutsche Gesellschaft für zerstörungsfreie Prüfung e. V. (Berlin) - Thermographie-Kolloquium, 2011. Berichtsband 130-CD, Vortrag 16

[5] FLIR, http://www.flir.com/cvs/eurasia/de/, FLIR Commercial Vision Systems (July 27, 2011)

[6] FLUKE, http://www.fluke.com/, Thermal Imaging - Smart View (July 27, 2011)

[7] C. Homma, "Investigation of the Mechanism and Technical Implementation of Acoustic Thermography", Dissertation, University of Saarbrücken, 2007

[8] M. Rothenfusser, C. Homma, "Acoustic Thermography: Vibrational Modes of Cracks and the Mechanisms of Heat Generation", Review of Quantitative Nondestructive Evaluation 24 (2005), pp. 624-631

[9] C. Homma, M. Rothenfusser, J. Baumann, R. E. Shannon, "Study of Heat Generation Mechanisms in Acoustic Thermography", Review of Quantitative Nondestructive Evaluation 25 (2006), pp. 566-573

[10] X. Han, Md. S. Ismal, "Finite element modeling of heating of cracks during sonic infrared imaging". In: J. Appl. Phys. 99, 2006 
[11] A. P. Bovsunovsky, "The mechanism of energy dissipation in the non-propagating fatigue cracks in metallic materials". In: Engineering Fracture Mechanics 71, 2004, No. 16-17, pp. 2271-81

[12] A. L. Audenino, C. Crupi, E. M. Zanetti, "Correlation between thermography and internal damping in metals". In: Int. Journal of Fatigue 25, 2003, No. 4, pp. 343-51 